\title{
Simple Approach to Traffic Update System
}

\author{
D Karmaker \\ Computer Vision Lab \\ Department of Computer \\ Science \\ American International \\ University-Bangladesh
}

\author{
M S U Miah \\ Computer Vision Lab \\ Department of Computer \\ Science \\ American International \\ University-Bangladesh
}

\author{
M A Imran \\ Computer Vision Lab \\ Department of Computer \\ Science \\ American International \\ University-Bangladesh
}

\author{
H Rahman \\ Computer Vision Lab \\ Department of Computer Science \\ American International University-Bangladesh
}

\author{
A Bhowmik \\ Computer Vision Lab \\ Department of Computer Science \\ American International University-Bangladesh
}

\begin{abstract}
Now a day's traffic jam is a very common problem in all the cities. Every day people spend valuable times uselessly because of traffic jam. We have proposed a system that came up with a solution which can reduce traffic jam dramatically. This is a web based application. People will just check the status of the route they are heading for whether the route consisting medium, heavy or less traffic. Moreover people can check all the alternative routes for the current traffic status that can help them taking decision for a traffic jam free route.
\end{abstract}

\section{Keywords}

Traffic Jam, Object Detection, Object Tracking, Kalman Filter, Masking, RPC.

\section{INTRODUCTION}

At present situation Traffic jam becomes a great problem for developing country. This paper approaches a simple solution to this problem. For this we developed a system. Anyone can check the condition of a road using internet from anywhere. For the system we need some pictures from every traffic signal. And these images will be processed in a web server. We can fetch the images from the traffic monitoring authorities. We have used image processing technologies for detecting the rush of a road. For every single there is a base image and this is an image of the empty state of that road. Images of the roads are fetched with a time period and compared with the base images. Then it calculates the percentage of traffic raises some events based on the calculation and sends it to the main server. Now if anyone wants to check the traffic of a specific road they simply have to go to the website then have to select the route. Then they can find where the road is busy or not. If busy is there any other route for their destination which is less busy. Thus they can reach their destination within shortest time.

\section{BACKGROUND STYDY}

A large number of researches have been done for traffic systems. One of the systems is VISATRAM [1]. This system takes two images from TV camera and compares those images and takes decision. Another system is TRIP (Traffic Research using Image Processing) [2].In this system the camera is placed in the road and take images .It compares the images using reference image. As a Result it returns threshold.

Another system to detect traffic is using sampling point to count the traffic [3]. Using two sample points to calculate the time and detecting the traffic. The Wide Area Detection System [4] is used for detecting, counting and measuring the speed of a vehicle. It reduces the image size. Camera and
Computer Aided Traffic Sensor using charge-coupled device [5].In this system this device is placed in the road and take pictures and only precedes 20 pixels per rail. It also worked in day and night. Kalman filters [6] system is capable of detecting and tracking many vehicles and give information of the vehicles. Another system is using the difference between consecutive two images to initialize a level which processed by a probabilistic motor [7]. It is classifying every pixel into this category: moving, still, uncovered and covered. In this system the position of vehicles occupy on the road in the current image. The limits for every object are established and calculate the speed of the vehicles.

As many cameras [9] are already been setup through the traffic authorities of the cities all we need the feeds from the network and calculate the necessary calculation to a server where all the requests by the users will be served. The position of the camera will be directly above the road's ending line, in the middle and facing the ground. The visual area of the camera will only cover the road that is under observation. The system needs an image template that represents the view of the road while no vehicles are under the camera. In this way of many image templates of different daytime will be taken as inputs and the system will learn the threshold values at different times of the day.
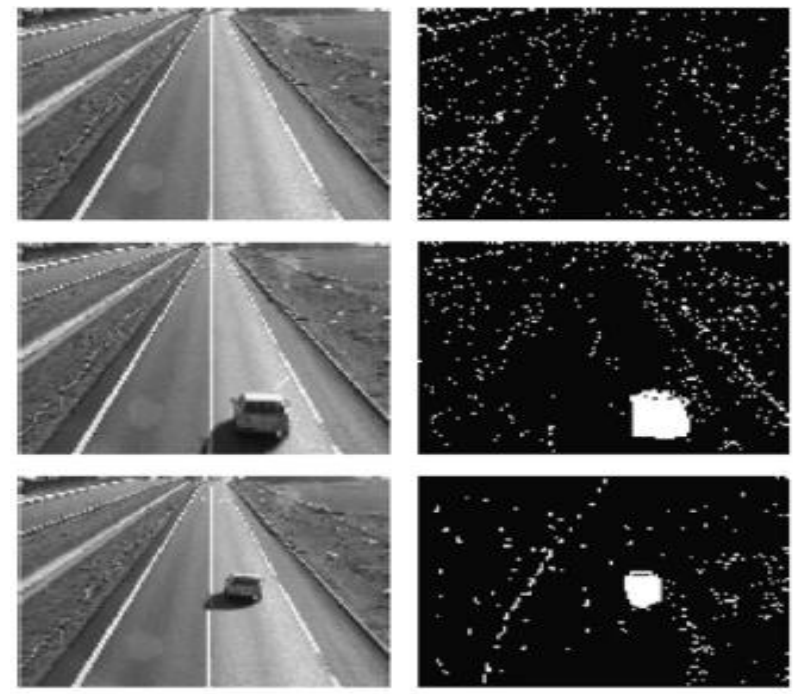

Fig 1: Empty road

After the calculating the threshold values the system will start taking images of the road for comparison purpose. The comparison process will take the input image and implement 
the threshold value then deduct the template image pixels from the current input image. Then we will only get the difference pixels containing vehicle figures. The system will then count the remaining pixels of the input image and calculate the difference of the two images in percentage (\%) value and store the data locally as a graph.

Any value that is less than $90 \%$ can be considered as there are stationary vehicles on the road. The graph analyzing process of the system will look for a short range of value lasting for more than 1 minute. If a range like that exist in the graph data then the system will start counting its starting time and wait for the ending of the continuous values then after the break down of the range the system will consider the time as a motionless event on the road. It can be a jam or a waiting time of the signal. If the continuous range is between fig: $3100 \%-$ $90 \%$ then there is no vehicles on the road. If short ranges are found repeatedly on the graph data then the road has a small amount of traffic jam with vehicles moving and stopping repeatedly. If no continuous range exists in the graph data and the mean of the values is less than 50\% (fig: 2) then it's a free road. The events found by analyzing the values and its duration will be sent to a remote server with the road identification number

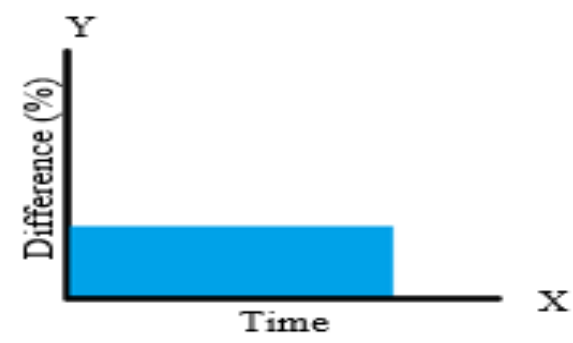

Fig 2: Vehicles standby (Jam)



Fig 3: Empty road

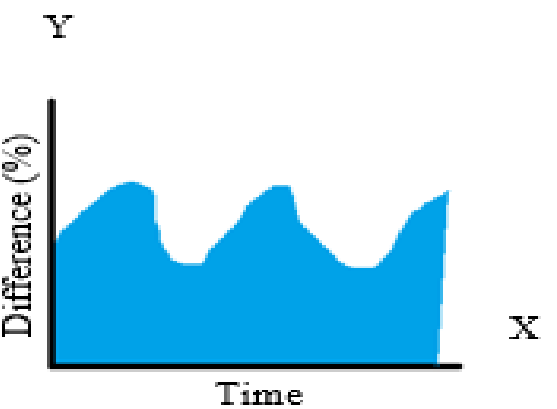

Fig 4: Fast moving vehicles (Less Traffic)

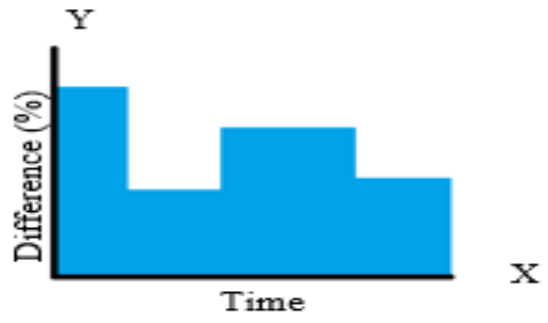

Fig 5: Very slow vehicles (More Traffic)

The remote server is the common database for all feeds of the network. It receives requests from the users that contains situation of the road and road identifying number. If visited, the index page will suggest a list of roads that has been already requested for and processed. The user can select the road and will be served with the longest waiting duration of that road.

\section{SYSTEM OVERVIEW}

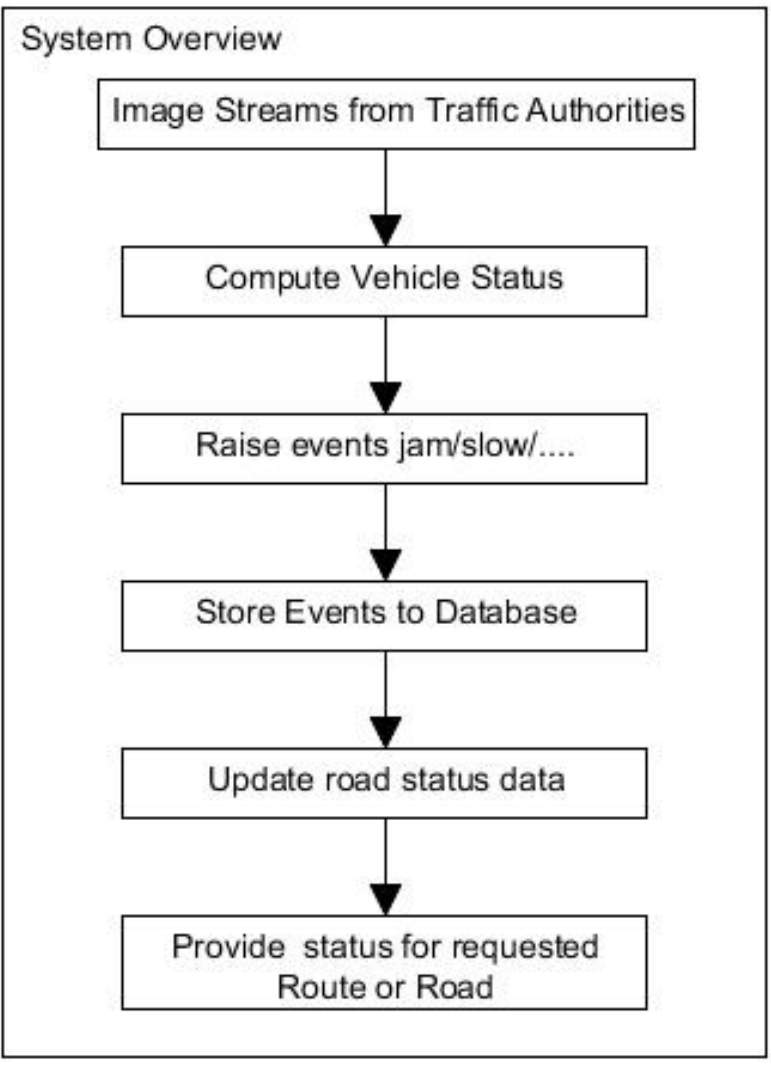

Fig 6: System Overview.

The system runs on the image streams that are coming from the traffic authorities. As the authority has already had a setup of traffic monitoring cameras on different roads, we only need the stream from those cameras. Then the system captures some images from the stream and does the necessary processing. After the processing, system generates the events [8] and stores to the database along with the road or route number. Then the status of the road is updated and user can get the updated status (fig: 6 ).

For the state generation process (fig:7) we need to pre compute empty road status for each road and this pre computed status is the base status or image is the base image for the computation. In the pre computation state we generate and store a threshold value for the empty road and later we 
compare it in runtime computation. In the runtime computation an image frame is taken and the threshold value is being compared with the pre computed one. If there is any change that means there might be some changes in the traffic statuses. Then an event is raised and stored into the database. This runtime computation is a continuous process and it continues unless there is no image stream.



Fig 7: System diagram of state generation

\section{ALGORITHM}

1. Initialize the threshold image value of empty road.

2. Take the image of current road.

3. Convert current road image into optimized black and white image, using appropriate threshold value.

4. Compare the optimized image with the empty road image.

5. Put the difference values into a graph like data structure got by the comparison of those two images.

6. By analyzing data of the graph, a new event will be raised or the old event remains unchanged.

7. The events will be specified as empty or jam or fast moving or slow moving.

8. After taking this result it will be sent that to the server along with the time duration and road identification number.

\section{PSEUDO CODE}

currentImg = CaptureNewImage ()

currentBitmap = ConvertToBitmap (currentImg)

bgImg = GetBackgroundAtCurrentTime()

FOR $i=\theta$ TO Width(currentImg) DO

FOR $j=0$ TO Height (currentImg) DO

IF $\operatorname{currentImg}(i, j)=\operatorname{bgImg}(i, j)$

INCRESE match ENDIF

ENDFOR

ENDFOR

diff $=($ match / (height*width $)) * 100$

graph $=$ AddNodeToGraph (diff)

curEvent $=$ CheckEvent (graph)

PostToServer(curEvent, road, Duration(curEvent))

\section{PERFORMANCE ANALYSIS}

We tested different images from different roads to analyze the correctness of the system. We have tested with 150 different images from different roads. We have acquired $87.33 \%$ of correctness from the images we have provided.

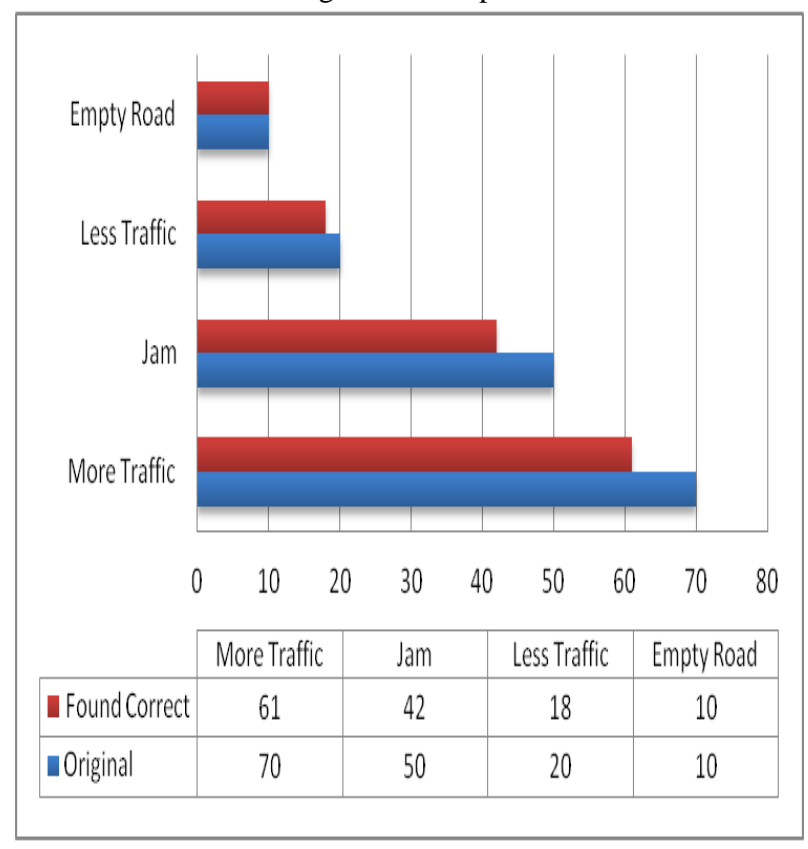

Fig 8: Performance analysis with each event. 




Fig 9: Overview of the performance analysis.

\section{CONCLUSION}

At the current situation we are trying to provide a system to mass users by which they can learn the status of the vehicle loads of different roads so that they can choose between alternate routes to reach their destinations. This automated system will automatically update the statuses of the different roads and it will help maintaining traffic loads equally to the alternate roads.

\section{REFERENCES}

[1] Zhu, Z., Xu, G., Yang, B., Shi, D., \& Lin, X. (2000). VISATRAM: A real time vision system for automatic traffic.
[2] Dickinson, K. W., \& Wan, C. L. (1989). Road traffic monitoring using the TRIP II system.

[3] Takaba, S., Sakauchi, M., Kaneko, T., Hwang, B. W., \& Sekine, T. (1982).Measurement of traffic flow using realtime processing of moving pictures.

[4] Inigo, R. M. (1989). Application of machine vision to traffic monitoring and control.

[5] Ali, A. T., \& Dagless, E. L. (1991). Computer visionaided road traffic monitoring.

[6] Koller, D., Weber, J., \& Malik, J. (1994). Robust multiple car tracking with occlusion reasoning.

[7] Barni, M., Bartolini, F., Capellini, V., Lambardi, F., \& Piva, A. (1998).Fuzzy motion detection for highway traffic control.

[8] M A U Rahman, M S U Miah, M A Fahad and D Karmaker, "SHIMPG: Simple human interaction with machine using Physical Gesture" Control Automation Robotics \& Vision (ICARCV), 2014 IEEE. [Online]. Available:

http://ieeexplore.ieee.org/xpl/articleDetails.jsp?arnumber $=7064322$.

[9] D karmaker, H Rahman, M S Rahaman, M S Rahman, "Global Motion tracking with six parameter model", ARPN Journal of Systems and Software,Volume 1 No. 5, 2011. [Online]. Available: http://scientificjournals.org/journalofsystemsandsoftware/archive/vol1n 05/vol1no5_3.pdf. 